\title{
Is it Time to Test Metformin in Breast Cancer Clinical Trials?
}

\author{
Massimiliano Cazzaniga, ${ }^{1}$ Bernardo Bonanni, ${ }^{1}$ Aliana Guerrieri-Gonzaga, ${ }^{1}$ \\ and Andrea Decensi ${ }^{2}$ \\ ${ }^{1}$ Division of Cancer Prevention and Genetics, European Institute of Oncology, Milan, Italy and ${ }^{2}$ Division of \\ Medical Oncology, Ospedali Galliera, Genova, Italy
}

\begin{abstract}
Several studies have identified an increased risk of cancer in type 2 diabetic patients and this is in accordance with the hypothesis that increased insulin levels might promote cancer. Thus, there is a great interest in exploring the possibility that antidiabetic therapies lowering insulin levels could decrease cancer incidence or cancer-related mortality. Recent observational studies have shown that metformin, an oral safe and well-tolerated insulin-sensitizer antidiabetic drug, has been associated with reduced cancer risk. Recently, several preclinical studies have evaluated the effect of metformin in vivo on nude mice and showed a significant reduction of both breast epithelial cell proliferation and protein synthesis. Further investigations in the clinical setting are well-supported by the
\end{abstract}

promising results obtained thus far. At the European Institute of Oncology, the Division of Cancer Prevention and Genetics is planning to conduct a clinical trial to evaluate the activity of metformin on tumor cell proliferation in breast cancer patients undergoing surgery. It will be a presurgical randomized, double blind, placebo-controlled phase II biomarker trial: 100 histologically confirmed breast cancer patients will be randomly assigned to metformin (850 $\mathrm{mg}$ twice/daily) or placebo for $28+7$ days till surgery to assess drug activity on tumor proliferation, as measured by Ki-67. The confirmation of the efficacy of metformin on cancer cell proliferation may lead the way to larger chemoprevention clinical trials. (Cancer Epidemiol Biomarkers Prev 2009;18(3):701-5)

\section{Introduction}

Despite major diagnostic and therapeutic innovations, treatment effect on breast cancer-related morbidity and mortality is still limited mainly because of the incomplete knowledge of the disease biology. Major research efforts continue to focus on identifying new agents able to treat or prevent breast carcinoma.

In the last two decades, there has been increasing evidence that physiologic concentrations of insulin may play a clinically important role in breast cancer development. A recent meta-analysis (1) has shown an increased risk of this disease in women with diabetes. In particular, the meta-analysis of 20 studies (5 case-control and 15 cohort studies) confirmed that diabetes was related with an increased risk of several kind of tumors and the combined results of 10 selected trials found that a high level of insulin was associated with a $13 \%$ and $25 \%$ increased risk of breast carcinoma in case-control and cohort studies, respectively. Moreover, it seems that insulin increased breast cancer recurrence and death $(2,3)$. Although the mechanism by which insulin influences breast carcinogenesis is still under investigation, it seems to activate several proliferative and antiapoptotic events through its own receptor (4). The

\footnotetext{
Received 9/17/08; revised 11/26/08; accepted 12/12/08; published OnlineFirst 2/24/09.

Requests for reprints: Massimiliano Cazzaniga, Division of Cancer Prevention and Genetics, European Institute of Oncology, Via Ripamonti 435, 20141, Milan, Italy. Phone: 39-02-57489861; Fax: 39-02-94379225. E-mail: massimiliano.cazzaniga@ieo.it Copyright $\odot 2009$ American Association for Cancer Research. doi:10.1158/1055-9965.EPI-08-0871
}

evidence that insulin influences breast carcinogenesis is extremely important because it raises the possibility of adopting insulin reduction for therapeutic and chemopreventive purposes in breast cancer.

Recent interest has been focused on metformin, a biguanide derivative, currently approved for the treatment of non insulin-dependent diabetes mellitus, and an insulin-sensitizing agent with potent antihyperglycemic properties. Its primary action is to inhibit hepatic glucose production, but it also increases the sensitivity of peripheral tissue to insulin (5). Thanks to these properties, metformin is an orally administered drug used to lower blood glucose concentration in patients with noninsulin-dependent diabetes mellitus and metabolic syndrome (6). Moreover, insulin resistence is one of the main features of the Polycystic Ovary Syndrome (PCOS), and the increase in insulin sensitivity metformin induces is used as PCOS therapy. PCOS is a disease characterized by the presence of chronic oligo-ovulation or anovulation, androgen excess, and polycystic ovaries, and is often associated with important metabolic derangements as diabetes mellitus and metabolic syndrome $(7,8)$.

\section{Metformin and Cancer}

Several studies have identified an increased risk of cancer in type 2 diabetes patients $(9,10)$ according to the hypothesis that increased insulin levels might promote cancer. Is indeed of great interest to explore the possibility that antidiabetic therapies (lowering 


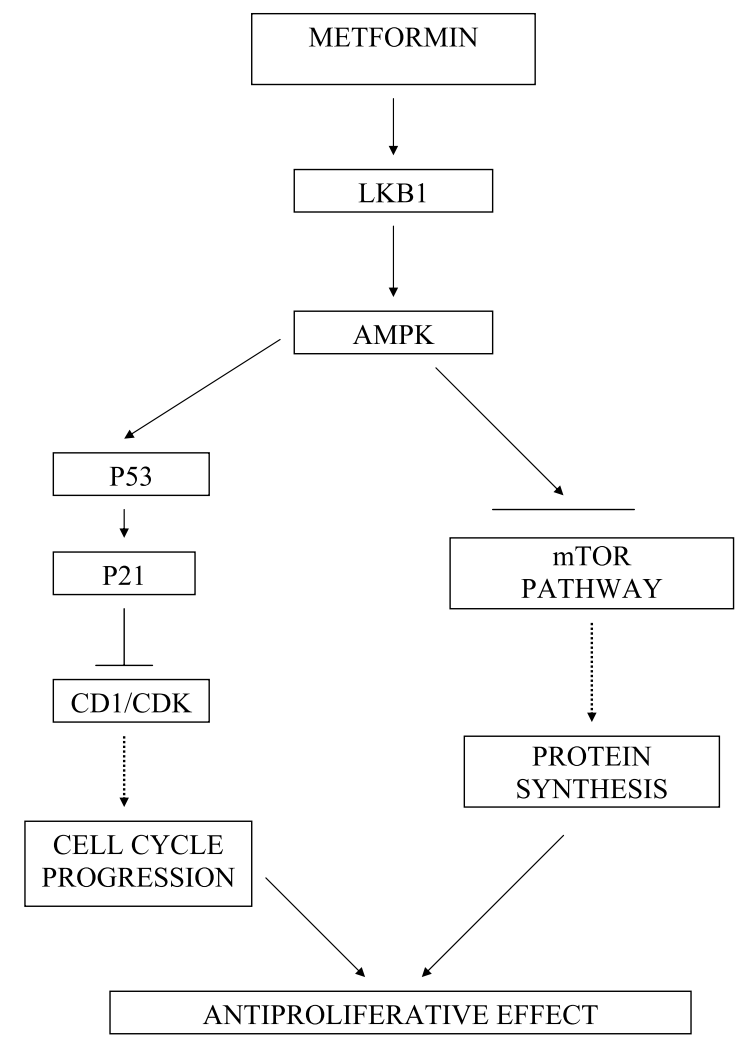

Figure 1. Complete mechanism of action of metformin on AMPK and proliferation.

insulin levels) could decrease cancer incidence or cancerrelated mortality.

Recent observational studies have shown that metformin treatment has been associated with reduced cancer risk. In particular, Evans et al. (11) observed that diabetic patients treated with metformin had a lower incidence of any kind of cancer when compared with patients on other treatments. Interestingly, this effect seemed related also to breast carcinoma and seems to improve with higher doses. Another observational study (12) involving $>10,000$ diabetic patients treated with metformin or other antidiabetic agents showed a lower cancer-related mortality rate in the metformin group when compared with other drugs, such as sulfonylureas or insulin.

Although the mechanism of action of metformin is still under investigation to determine whether these observations on the antineoplastic action of metformin might be related to the direct action of this drug on cancer cells or to its indirect effect, such as insulin level reduction. At present, both these biological pathways seem related to its potential anticancer activity. In particular, it is biologically plausible that there may be a direct effect due to the influence of the physiologic levels of insulin on the proliferation of breast cancer cells, considering the uniform expression on breast carcinoma of its receptor. An indirect influence is also possible: metformin can suppress protein synthesis and cell proliferation with AMP-dependent protein kinase (AMPK) activation.

\section{The Mechanism of Action of Metformin}

As regards glucose homeostasis properties, the molecular mechanism of metformin has been studied in different tissues, such as muscle and fatty tissues and liver. Although, the effect on epithelial cells remains unclear. This aspect should be clarified for there is substantial evidence that the mechanism of action may involve AMPK activation (an AMP-activated protein kinase serving as an energy sensor) by means of an LKB1-dependent mechanism (13). LKB1 is a tumor suppressor gene with relevance to epithelial neoplasia; in particular, its activity loss is frequently associated with a syndrome, named Peutz-Jeghers, characterized by several gastrointestinal polyps and also by a significantly increased risk of various epithelial cancers, including breast cancer (14).

According to several published studies, AMPK activation strongly suppresses cell proliferation in both malignant and nonmalignant cells. These AMPK actions seem to be mediated by multiple mechanisms: cell cycle regulation and protein synthesis inhibition.

The first pathway is mediated by an up-regulation of the p53-p21 axis, the reduction of Cyclin D1 levels with the consequent inhibition of the correspondent cyclindependent kinases, eventually causing an antiproliferative effects by the $G_{1}$ cell cycle arrest. The second pathway involves the mammalian target of rapamycin. The complete mechanism of action on AMPK and proliferation of metformin is summarized in Fig. 1.

The hypothesis that metformin controls cellular proliferation as well as protein synthesis in epithelial cells has been shown in recent preclinical studies.

\section{Potential Adverse Effects}

In its clinical use, metformin has shown a good safety profile and has resulted well-tolerated in patients. The main limited side effect was gastrointestinal distress, such as nausea and diarrhea, and it was transient. Only few cases required discontinuation of the drug. Metformin may also promote hirsuitism and can cause vitamin B12 malabsorption in long-term therapy (5). No teratogenic effects or adverse fetal outcomes were reported. The only potential major adverse event in metformin therapy is lactic acidosis. This condition is rare and is primarily confined in patients with concomitant renal and hepatic disorders (5).

Despite metformin has been used for many years in patients with type 2 diabetes and presented preclinical data on its potential as an anticancer agent are promising, there are no data regarding the potential short- and longterm effect of this drug in nondiabetic patients or affected by PCOS.

\section{Preclinical Studies}

Sahra et al. (15) have recently investigated the effect of metformin on human prostate cancer cell proliferation both in vitro and in vivo. The action of different doses of the drug on three prostate cancer cell lines compared with one normal cell line. The authors have found out that metformin can lead to a dose-dependent strong 
inhibition of cell proliferation in all prostate cell lines, blocking cell cycle by means of a strong decrease of cyclin D1 protein level. On the contrary, a moderate effect on normal cells has been noticed. These results suggest that metformin may specifically affect cancer cell proliferation compared with that of normal cells. Moreover, researchers have detected that metformin is able to reduce proliferation in colon and breast cancer cell lines. To confirm these data in vivo, metformin was either i.p. injected in nude mice or orally administred with water. This resulted in a tumor growth inhibition of $35 \%$ and $55 \%$, respectively.

These data confirm the results published by Zakikhani et al. (16), according to which the AMPK pathway activation by means of metformin was observed in epithelial cells, together with a reduced proliferation and a general protein synthesis reduction. To evaluate the effect of different doses of the drug on proliferation, researchers have in particular analyzed the effect of this drug in vitro on a series of transformed and untransformed epithelial cell lines (breast, ovary, and prostate). Metformin was shown to act as a growth inhibitor rather than an insulin sensitizer, above all for MCF-7 human breast cancer cells. Moreover, Zakikhani found out that cell line exposure to the growth inhibitory concentrations of metformin can lead to a general decline in protein synthesis and ultimately to block both growth and proliferation. Interestingly, metformin is unlikely to directly affect those cancers inhibiting biallelic loss of LKB1 function. These data confirm that the effect of metformin on AMPK activation-mediated pathway require LKB1 suppressor gene activation.

After the results gathered on breast cancer cell lines, another study has tried to analyze the effect of metformin in different types of breast cancer cell lines, with particular attention to the hormonal characteristics of the disease. Phoenix et al. (17) confirmed that AMPK stimulation by metformin results in a significant repression of cell proliferation. She also found out that this effect was different in human breast cancer cell lines if related to either positive or negative estrogen receptors (ER). She detected complete cell growth repression in two ER-positive cell lines, although only a partial inhibition in ER-negative phenotypes. These data suggest that although both positive and negative ER breast cancer cells reduce cell growth under metformin treatment, the ER-negative cells are not as sensitive as the ER-positive ones. Moreover, researchers found a significant increase in vascular endothelial

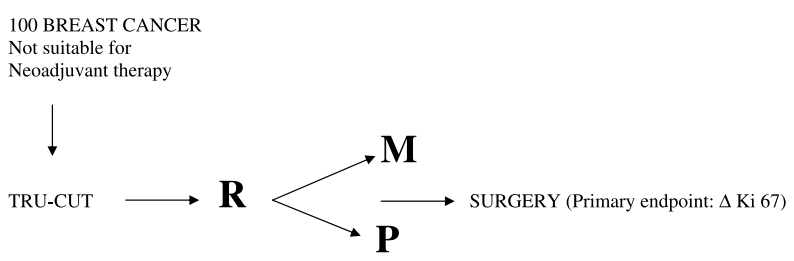

Stratification according to ER status

$\mathrm{R}=$ randomization; $\mathrm{M}=$ metformin $(850 \mathrm{mg} / \mathrm{die}$ for 3 days followed by $850 \mathrm{mg}$ twice daily from days 4 to $28 \pm 7$ ); $P=$ placebo

Figure 2. Design of presurgical metformin study. growth factor ER-negative cell lines. This is of paramount importance because the increase of metformin-mediated vascular endothelial growth factor may override its antiproliferative effect in this particular phenotype and apparently results in proangiogenic stimuli. This mechanism could facilitate tumor progression as shown in vivo in the same study. However, these preclinical data need to be further investigated in the clinical setting.

\section{Related Biomarkers}

In general, an appropriate biomarker should be able to define risks and to identify the early stages of carcinogenesis. In addition, biomarkers are also able to make drug development more rational, thereby optimizing preclinical and clinical efforts and delivering timely benefits to patients. In previous preclinical studies, the effect of metformin was shown on some of these biomarkers. Sahra et al. (15) have shown the direct effect of this drug on cyclin D1. The effect of metformin on other biomarkers involved in cell cycle regulation and proliferation should be further investigated. Moreover, the possibility to better understand metformin-mediated effect on serum estrogen endogenous hormones and, in particular, on their cellular receptors has been discussed. Metformin has been proven to have an effect on ovarian androgens through the reduction of the circulating levels of insulin (insulin up-regulates ovarian steroidogenesis; ref. 18), may decrease ovarian androgen levels through a decrease in luteinizing hormone, may diminish androgen production due to an increase in tyrosine activity in metformin-exposed ovarian cells, therefore leading to a decrease in steroidogenic enzyme activity.

The insulin-like growth factor system (IGF) and its components (IGF-I and IGF-II) are peptides, whose mitogenic and antiapoptotic effects influence the proliferation of various cell types, including normal and transformed epithelial breast cells (19). These biomarkers have been thoroughly investigated in selected women with PCOS. In general, patients present lower levels of IGF binding protein-I, leading to increased ovarian bioavailability of IGF-I. On the basis of these observations, clinical trials have been conducted to assess whether metformin affects IGF pathways or its binding proteins (20-23). Unfortunately, this issue remains controversial. The most accredited hypothesis is that the drug reduces IGF-I concentration by indirectly increasing its binding protein (IGF binding protein-I), and not directly affecting IGF-I.

According to Buzzai et al. (24), metformin can increase apoptosis and can therefore be selectively toxic in paired isogenic p53-deficient colon cancer cell lines. P53 could be another biomarker to investigate metformin's action in clinical studies.

\section{Purpose}

The promising results of observational and preclinical studies support further investigations on metformin in clinical settings and, in particular, in breast cancer 
treatment and prevention. Metformin could be investigated in two different settings:

- Presurgical model: to evaluate whether the use of different doses of metformin in early breast cancer preoperative settings can modulate tissue and serum biomarkers triggering tumor proliferation biomarkers. In this kind of studies, patients with a diagnostic core biopsy for intraductal neoplasia or invasive cancer are randomized to one of several doses in the time interval between biopsy and surgery (generally 4-6 wk). These phase II trials usually focus on establishing the lowest dose at which the drug modulates the risk and/or the mechanism of action of the biomarker. In spite of the significant problems with these presurgical models (i.e., difficulties of accrual, significant variations of the value of the biomarkers in different parts of the lesion), these approaches showed good feasibility and could serve as a spingboard for future investigations on the presence of these biomarkers in healthy high risk women.

- Blind, randomized, placebo-controlled phase III study, in selected cohort of healthy high-risk women, harnessing the property of metformin to reduce or modulate hormonal status, in particular to assess its ability to decrease androgen levels, which are a recognized risk factor for breast cancer.

At present, in breast cancer settings, the substantial absence of clinical data on safety, on minimal dose efficacy, and on the effects on biomarkers, discourages an immediate phase III study. On the other hand, such lack of data highlights the pressing need for biomarker phase I to II studies. Our Division of Cancer Prevention and Genetics is going to start a presurgical study on women with histologically confirmed breast cancer. Our goal is to evaluate the real activity of metformin on tumor cell proliferation. We also aim to test the differences in the percentage change reduction in Ki-67 proliferation antigen evaluated before and after treatment and compare the data with placebo group. Secondary end points of the study will be to determine the interaction effect of metformin on Ki-67 by ER status as well as to observe the effect of this agent on some tissue biomarkers (Cyclin D1, p53, vascular endothelial growth factor).

Women with palpable breast cancer eligible for a trucut biopsy and not suitable for neoadjuvant therapy will be randomized and they will be given either metformin or placebo. The population target will be 100 women with histologically confirmed primary breast cancer, stage I/II/IIIa (T $\geq 1 \mathrm{~cm}, \mathrm{~N} 0-1, \mathrm{M} 0$ ), not suitable for neoadjuvant therapy. After biopsy, patients will be randomly assigned to either metformin or placebo tablets $850 \mathrm{mg}$ daily from day 1 to day 3 (to exclude treatment side effects), to be increased to $850 \mathrm{mg}$ twice a day from day 4 to day $28 \pm 7$. The complete design of our study is summarized in Fig. 2.

Given the short time of exposure to treatment, both ER-positive and ER-negative patients will be included to evaluate the potential different effect of the drug according to ER status as observed in the preclinical setting.

\section{Conclusions}

Metformin is an insulin-sensitizer agent, widely used to treat type 2 diabetes with a relatively favorable toxicity profile. In epithelial cells, metformin-induced AMP kinase activation has been shown to activate growth inhibitory and protein synthesis pathways. Moreover, it may have indirect antiproliferative properties through its insulin-lowering action, as suggested by population studies reporting an unexpectedly lower cancer incidence rate in diabetic patients treated with metformin compared with other therapies. As a consequence, insulin-lowering approaches by means of pharmacologic therapies may have potential in cancer prevention and prognosis.

Further studies are necessary to clarify the role of this agent in breast cancer prevention and therapy. Its favorable toxicity profile, its relative low cost, and evidence of its antiproliferative activity detected both in vitro and in vivo in preclinical studies should stimulate researchers to carry out further clinical trials on this agent.

\section{Disclosure of Potential Conflicts of Interest}

No potential conflicts of interest were disclosed.

\section{References}

1. Larsson SC, Mantzoros CS, Wolk A. Diabetes mellitus and risk of breast cancer. A meta-analysis. Int J Cancer 2007;121:856-62.

2. Goodwin PJ, Ennis M, Pritchard KI, et al. Fasting insulin and outcome in early stage breast cancer: results of prospective cohort study. J Clin Oncol 2002;20:42-51.

3. Pollak MN, Chapman JW, Sheperd L, et al. Insulin resistence, estimated by serum C-peptide level, is associated with reduced event-free survival for postmenopausal women in NCIC CTG MA.14 adjuvant breast cancer trial. J Clin Oncol 2006;24:524.

4. Papa V, Belfiore A. Insulin receptors in breast cancer: biological and clinical role. Endocrinol Invest 1996;19:324-33.

5. Bailey CJ, Turner RC. Metformin. N Engl J Med 1996;334:574-9.

6. Bailey CJ. Biguanides and NIDDM. Diabetes Care 1992;15:755-72.

7. Nestler JE. Metformin for the treatment of the polycystic ovary syndrome. N Engl J Med 2008;358:47-54.

8. Cheang T, Sharma ST, Nestler JE. Is metformin a primary ovulatory agent in patients with polycystic ovary syndrome. Gynecol Endocrinol 2006;22:595-604.

9. Hsu IR, Kim SP, Kabir M, et al. Metabolic syndrome, hyperinsulinemia and cancer. Am J Clin Nutr 2007;86:867-71.

10. Xue F, Michels KB. Diabetes, metabolic syndrome and breast cancer: a review of the current evidence. Am J Clin Nutr 2007;86:823-35.

11. Evans JM, Donnelly LA, Emslie-Smith AM, et al. Metformin and reduced risk of cancer in diabetic patients. BMJ 2005;330:1304-5.

12. Bowker SL, Majumdar SR, Veugelers $P$, et al. Increased cancerrelated mortality for patients with type 2 diabetes who use sulfonylureas or insulin. Diabetes Care 2006;29:254-8.

13. Zhou G, Myers R, Li Y, et al. Role of AMP-activated protein kinase in mechanism of metformin action. J Clin Invest 2001;108:1167-74.

14. Giardiello FM, Brensinger JD, Tersmette AC, et al. Very high risk of cancer in familial Peutz-Jeghers syndrome. Gastroenterology 2000; 119:1447-53.

15. Sahra IB, Laurent $K$, Loubat $A$, et al. The antidiabetic drug metformin exerts an antitumoral effect in vitro and in vivo through a decrease of cyclin D1 level. Oncogene 2008;27:3576-86.

16. Zakikhani M, Dowling R, Fantus IG, et al. Metformin is an AMP kinase-dependent growth inhibitor for breast cancer cells. Cancer Res 2006;66:10269-73.

17. Phoenix KN, Vumbaca F, Claffey KP. Therapeutic metformin/AMPK activation promotes the angiogenic phenotype in the ER $\alpha$ negative MDA-MB-435 breast cancer model. Breast Cancer Res Treat 2008 [epub ahead of print].

18. Ehrmann DA, Cavaghan MK, Imperial J, et al. Effects of metformin on insulin secretion, insulin action, and ovarian steroidogenesis in 
women with polycystic ovary syndrome. J Clin Endocrinol Metab 1997;82:524-30.

19. Sachdev D, Yee D. The IGF system and breast cancer. Endocr Relat Cancer 2001;8:197-209.

20. De Leo V, La Marca A, Orvieto R, et al. Effect of metformin on insulinlike growth factor (IGF) I and IGF-binding protein I in polycystic ovary syndrome. J Clin Endocrinol Metab 2000;85:1598-600.

21. Berker B, Emral R, Demirel C, et al. Increased insulin-like growth factor-I levels in women with polycystic ovary syndrome, and beneficial effects of metformin therapy. Gynecol Endocrinol 2004;19: $125-33$.
22. Seibel SA, Chou KH, Capp E, et al. Effect of metformin on IGF-I and IGFBP-I levels in obese patients with polycystic ovary syndrome. Eur J Obstet Gynecol Reprod Biol 2007 [epub ahead of print].

23. Kowalska I, Kinalski M, Straczkowski M, et al. Insulin, leptin, IGF-I and insulin-dependent protein concentrations after insulin-sensitizing therapy in obese women with polycystic ovary syndrome. Eur J Endocrinol 2001;144:509-15.

24. Buzzai M, Jones RG, Amaravadi RK, et al. Systemic treatment with the antidiabetic drug metformin selectively impairs p53-deficient tumor cell growth. Cancer Res 2007;67:6745-52. 


\section{Cancer Epidemiology, \\ Biomarkers \& Prevention}

\section{Is it Time to Test Metformin in Breast Cancer Clinical Trials?}

Massimiliano Cazzaniga, Bernardo Bonanni, Aliana Guerrieri-Gonzaga, et al.

Cancer Epidemiol Biomarkers Prev 2009;18:701-705.

Updated version Access the most recent version of this article at:

http://cebp.aacrjournals.org/content/18/3/701

Cited articles This article cites 22 articles, 8 of which you can access for free at:

http://cebp.aacrjournals.org/content/18/3/701.full\#ref-list-1

Citing articles This article has been cited by 25 HighWire-hosted articles. Access the articles at:

http://cebp.aacrjournals.org/content/18/3/701.full\#related-urls

E-mail alerts Sign up to receive free email-alerts related to this article or journal.

Reprints and To order reprints of this article or to subscribe to the journal, contact the AACR Publications

Subscriptions Department at pubs@aacr.org.

Permissions To request permission to re-use all or part of this article, contact the AACR Publications Department at permissions@aacr.org. 